\title{
REGENERAÇÃO NATURAL EM REMANESCENTES FLORESTAIS E ÁREAS REFLORESTADAS DA VÁRZEA DO RIO MOGI-GUAÇU, LUIZ ANTÔNIO - SP1
}

Assíria Maria Ferreira da Nóbrega ${ }^{2}$, Sérgio Valiengo Valeri ${ }^{3}$, Rinaldo César de Paula ${ }^{3}$ e Sérgio Adriano da Silva ${ }^{4}$

\begin{abstract}
RESUMO - O trabalho objetivou descrever e avaliar a estrutura da regeneração de espécies arbóreas em dois remanescentes naturais e em três áreas reflorestadas com espécies nativas e em um povoamento de Eucalyptus

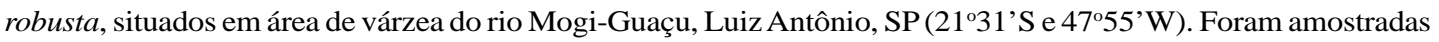
40 subparcelas de $2 \mathrm{~m}^{2}$ em cada remanescente natural e sub-bosque de eucalipto e 60 subparcelas de $3,5 \mathrm{~m}^{2}$ em cada área reflorestada. Foram amostrados todos os indivíduos arbóreos de regeneração com altura $\geq$ a 10 $\mathrm{cm}$ e diâmetro do caule até a altura do peito (DAP) $<5,0 \mathrm{~cm}$ e analisados separadamente, em quatro classes de altura, a diversidade florística, a regeneração natural ( $\mathrm{Rn} \%$ ), o valor de importância (VI) e a similaridade da regeneração com indivíduos de DAP $\geq 5 \mathrm{~cm}$. Foram identificados 1.990 indivíduos, pertencentes a 24 famílias, 46 gêneros e 51 espécies. Cabralea canjerana, Psidium cattleyanum, Nectandra megapotamica, Acacia polyphylla e Syzygium cumini estavam entre as espécies mais representadas nas quatro categorias de tamanho. O reflorestamento com espécies nativas em áreas degradadas da várzea do rio Mogi-Guaçu promoveu a regeneração natural com biodiversidade superior aos remanescentes naturais de florestas ciliares sob efeito de borda e contribuiu para com o processo de restauração de ecossistemas florestais. O povoamento de Eucalyptus robusta com cerca de 20 anos de idade favoreceu a regeneração de espécies climácicas e secundárias.
\end{abstract}

Palavras-chave: Fitossociologia, índice de diversidade e Eucalyptus robusta.

\section{NATURAL REGENERATION IN FOREST REMAINDERS AND REFORESTED AREAS OF “MOGI-GUACUU” RIVER FLOODPLAIN IN LUIZ ANTÔNIO COUNTY, SÃO PAULO, BRAZIL}

\begin{abstract}
The phytosociology of the woody stratum of both young regenerating individuals and seed banks are quality indicators of heterogeneous reforestations. The objetive of this research was to evaluate the structure of regeneration of the arboreal species in two natural remainders, in three areas reforested with native species and in a Eucalyptus robusta settlement located in a floodplain of Mogi-Guaçu river in Luiz, Antônio County $\left(21^{\circ} 31^{\prime} \mathrm{S}\right.$ and $\left.47^{\circ} 55^{\mathrm{W}} \mathrm{W}\right)$, São Paulo, Brazil. Sampling was collected in 40 subplots of $2 \mathrm{~m}^{2}$ in of each natural remainder and each eucalyptus sub-forest, as well as 60 subplots of $3.5 \mathrm{~m}^{2}$ in each area reforested. All individuals with height $\geq 10 \mathrm{~cm}$ and diameter at breast height $(\mathrm{DBH})<5.0 \mathrm{~cm}$ were measured and analyzed separately in four height classes according to floristic diversity, similarity, density, frequency, dominance, importance value and natural regeneration. A total of 2,010 individuals were identified, belonging to 24 families, 46 genera and 51 species. The species with greater importance values were Cabralea canjerana, Psidium cattleyanum, Nectandra megapotamica, Acacia polyphylla and Syzygium cumini, well represented in the four height classes. Reforestation with native species in a degraded Mogi-Guaçu River floodplain promoted natural regeneration with more biodiversity than in natural remainders submitted to border effect, and contributed to the forest ecosystem restoration process. The 20-year-old Eucalyptus robusta plantation promoted the regeneration of climax and secondary species.
\end{abstract}

Keywords: Phytosociology, diversity index and Eucalyptus robusta.

\footnotetext{
${ }^{1}$ Recebido em 03.02.2007 e aceito para publicação em 22.08.2008.

${ }^{2}$ Departamento de Engenharia Florestal da Universidade Federal de Campina Grande (UFCG). E-mail:〈amfnobrega@ig.com.br>

${ }^{3}$ UNESP- Universidade Estadual Paulista, Campus de Jaboticabal, Departamento de Produção Vegetal. 14884-900 JaboticabalSP. Bolsista do CNPq.E-mail:<valeri@fcav.unesp.br>e <rcpaula@fcav.unesp.br>.

${ }^{4}$ Setor de Pesquisa e Gestão Ambiental da International Paper do Brasil. E.mail:<sergio.silva@ vcp.com.br>.
} 


\section{INTRODUÇÃO}

O processo de fragmentação florestal de origem antrópica no Brasil iniciou a partir de 1500, com a conquista deste continente pelos europeus. Inicialmente, em decorrência da extração de madeiras como o paubrasil para o comércio ou, simplesmente, a derrubada da floresta para fins energéticos e implantação de infraestrutura urbana. Depois disso, os principais fatores da fragmentação foram os ciclos econômicos, como a conquista de terras para a agricultura, mineração e construção de barragens de rios (FISZON et al., 2003).

Nas áreas de alta densidade populacional, restaram fragmentos florestais isolados e florestas ciliares degradados por ações antrópicas e pelos efeitos de borda que favorecem o crescimento de espécies pioneiras. Entre essas espécies, destacam-se espécies arbóreas de crescimento rápido, lianas e gramíneas. Assim, esses ecossistemas florestais alterados pela ação antrópica apresentam baixa diversidade de espécies e desequilíbrio na proporção dos três grupos ecológicos sucessionais dos indivíduos arbóreos, a saber: das pioneiras, das secundárias e das climácicas (SCARIOT et al., 2003).

Entre as técnicas mais importantes para a recuperação de ecossistemas florestais degradados, podem ser citadas: a revegetação, que seleciona espécies dos três grupos ecológicos; a escolha de espaçamento e arranjo de plantio (blocos e quincôncios, entre outros), a condução da regeneração natural e os plantios de enriquecimento (BARBOSA, 2006).

Para que ocorra regeneração natural em uma área virtualmente degradada ou em processo de degradação, são necessárias algumas condições, como o cessar dos processos causadores da degradação, entre eles pastoreio e incêndios, a existência de fonte de propágulos (banco de sementes do solo, chuva de sementes), presença de dispersores, boas condições microclimáticas e edáficas, ausência de predadores e agentes antrópicos, para o estabelecimento e ocorrência do ciclo de vida completo das plântulas (FARIA et al., 2001).

O entendimento dos processos de regeneração natural é importante para o sucesso do manejo das florestas (DANIEL e JANKAUSKIS, 1989). Os estudos de regeneração natural, de dinâmica dos processos de sucessão ecológica e do banco de sementes são fundamentais para o entendimento do estabelecimento e evolução de um ecossistema florestal (NAPPO et al., 1999). Para Rodrigues (1999), a regeneração natural pode ser avaliada por meio de um levantamento florístico e, ou, estrutural (densidade, dominância e freqüência por espécie), de plântulas ou de indivíduos jovens, usando-se qualquer método fitossociológico.

No Estado de São Paulo, existem 4.372 fragmentos florestais menores do que 10 ha contra 47 fragmentos maiores do que 400 ha, e a maioria está sob efeito de borda (KRONKA et al., 2005). Segundo Barbosa (2000), estima-se que o Estado possui cerca de 600.000 ha de áreas de preservação permanentes degradas e situadas ao longo dos cursos d'água, que precisam ser reflorestadas. Os estudos de regeneração natural devem ser usados como indicadores da capacidade de resiliência de ecossistemas florestais e da qualidade dos reflorestamentos heterogêneos, com base em Barbosa (2006).

Este trabalho teve como objetivo avaliar a regeneração natural em remanescentes naturais e áreas reflorestadas com espécies nativas e em um povoamento de Eucalyptus robusta, situados em uma várzea do rio Mogi-Guaçu, a fim de verificar se as espécies dos remanescentes naturais estão se regenerando nas áreas reflorestadas.

\section{MATERIAL E MÉTODOS}

\subsection{Descrição do meio físico}

Este trabalho foi realizado na Fazenda Guatapará, pertencente à companhia International Paper do Brasil, localizada no Município de Luiz Antônio, SP (21 $31^{\circ}$ S e $\left.47^{\circ} 55^{\prime} \mathrm{W}\right)$.

A topografia da região é plana, com altitude média de 500 m. Conforme a classificação de Köppen, o clima da região é do tipo Cwa subtropical (quente de inverno seco). A área de estudo apresenta duas fitofisionomias, com base em Leitão-Filho (1982). A primeira é uma floresta latifoliada semicaducifolia com inundação esporádica (mata ciliar), localizada nas proximidades do rio MogiGuaçu e denominada ripária RIP1 (5,89 ha). Esse rio tem por característica mudar periodicamente de lugar e, provavelmente, no passado seu trajeto deveria ter sido ao lado da Mata Ciliar denominada ripária RIP2 (16,41 ha). A segunda fitofisionomia é uma floresta latifoliada higrófila (mata de brejo). $\mathrm{Na}$ área de estudo, o solo predominante é o Neossolo Flúvico Distrófico (EMBRAPA, 1999). 


\subsection{Histórico das áreas de estudo}

Há mais de 20 anos, parte da Mata Ciliar paludosa da várzea, inundável apenas no período das chuvas, foi substituída pelo cultivo de arroz e, posteriormente, abandonada, favorecendo o estabelecimento de diversas gramíneas como capim-jaraguá (Hyparrhenia rufa), capim-colonião (Panicum maximum), braquiária (Brachiaria decumbens) e capim-navalha (Echinochloa polystachya), entre outras. A Guatapará Florestal efetuou o plantio de um talhão de Eucalyptus robusta no interior da várzea de 3,46 ha, entre as florestas ripárias RIP1 e RIP2, em 1983, no espaçamento de plantio 3 m x 2 $\mathrm{m}$. Durante o período de dezembro de 1990 a maio de 1992, a Votorantim Celulose e Papel efetuou três reflorestamentos com 41 espécies nativas em cada um, denominados R1 (16,1 ha), R2 (21,8 ha) e R3 (21,9 ha), tendo sido adotado o espaçamento médio de $3 \mathrm{~m} \mathrm{x}$ $2 \mathrm{~m}$. Também, foram plantadas mudas de jambolão (Syzygium cumini) nos três reflorestamentos, além de cássia-nodosa (Cassia nodosa) em R1, mangueira (Mangifera indica) em R2 e R3 e tento-carolina (Adenanthera pavonina) em R3.

\subsection{Amostragem}

A amostragem dos indivíduos em regeneração foi realizada nos dois remanescentes naturais (RIP1 e RIP2), em três áreas de reflorestamento com espécies nativas (R1, R2 e R3) e em um povoamento de Eucalyptus robusta com idade de 19 anos e plantado no espaçamento $3 \mathrm{~m} \times 2 \mathrm{~m}$.

Foram registrados os indivíduos vivos de espécies arbóreas com diâmetro à altura do peito (a 1,30 m do nível do solo) - DAP $<5,0 \mathrm{~cm}$ e que se encontravam regenerando naturalmente no sub-bosque das áreas de amostragens. Para a avaliação da similaridade florística de Jaccard entre o estrato arbóreo (árvores com DAP $\geq 5,0 \mathrm{~cm}$ ) e o sub-bosque em regeneração, foram usados os dados disponibilizados por Nóbrega (2003).

As amostragens foram realizadas em 20 parcelas de $10 \mathrm{~m}$ x $10 \mathrm{~m}$ em cada área, entre julho e setembro de 2002. Foram usadas duas subparcelas de $2 \mathrm{~m}$ x 1 $\mathrm{m}$ nos remanescentes naturais e sub-bosque de eucalipto e nas áreas de reflorestamento heterogêneo $(\mathrm{R} 1, \mathrm{R} 2$ e R3) três faixas de 3,5 m x 1,0 m, todas locadas aleatoriamente, por meio de sorteio, dentro de cada parcela. Em seguida, foram amostrados todos os indivíduos de espécies arbóreas com altura superior a $10 \mathrm{~cm}$, os quais foram classificados em quatro classes de tamanho, conforme Finol (1971), em: Ct I - indivíduos com altura de $0,10-0,50 \mathrm{~m}$; Ct II - indivíduos com altura de 0,51 - 1,0 m; Ct III - indivíduos com altura de 1,0-2,0 m; e Ct IV - indivíduos com altura superior a 2,0 m e DAP $<5,0 \mathrm{~cm}$.

O diâmetro do caule foi medido a $7 \mathrm{~cm}$ de altura do solo, para evitar a região de engrossamento irregular que ocorre na parte inferior do caule das plantas encontradas no local. A identificação taxonômica foi realizada com o auxílio de especialista do Herbário do Departamento de Botânica da Escola Superior de Agricultura “Luiz de Queiroz,” da Universidade de São Paulo.

\subsection{Parâmetros avaliados}

Para análise da regeneração natural, foram calculados os seguintes descritores: densidade, freqüência, classe de tamanho, abundância relativa, regeneração natural, valor importância (VI), índices de Shannon $\left(\mathrm{H}^{\prime}\right)$ e eqüabilidade $(\mathrm{J})$, conforme Carvalho (1984) e Caldato et al. (1996). A análise da estrutura vertical foi feita por meio da posição sociológica (CARVALHO, 1984). As famílias foram organizadas de acordo com a proposta da Angiosperm Phylogeny Group II (APGII) de 2003, com base em Souza e Lorenzi (2005). Quanto aos grupos ecológicos de sucessão, as espécies foram classificadas em pioneiras, secundárias e climácicas, de acordo com os critérios estabelecidos por Budowsky (1965) e com base nas características das espécies descritas por Lorenzi (1992), Lorenzi (1998) e Carvalho (2003).

\section{RESULTADOS E DISCUSSÃO}

Foram registrados 1990 indivíduos na composição florística da regeneração natural, sendo estes distribuídos em 46 gêneros, 24 famílias e 51 espécies. Das espécies registradas, 51 foram identificadas e duas não-identificadas (Quadro 1). Há uma variação entre os números de indivíduos observados por espécie, com alta predominância de Nectandra megapotamica, espécie climácica que apresenta alta capacidade de regeneração em ambientes sombreados do interior de Florestas Tropicais Semidecíduas, com 20,93\% do total de indivíduos amostrados, seguida de Cabralea canjerana, Pisidium cattleyanum, Acacia polyphylla, Inga striata, Cecropia hololeuca e Sebastiana commersoniana, todas representando $68 \%$ dos indivíduos observados (Quadro 1).

R. Árvore, Viçosa-MG, v.32, n.5, p.909-920, 2008 
Quadro 1 - Famílias, espécies, nomes regionais, número de indivíduos (N) e grupo ecológico (GE) observados na várzea do rio Mogi-Guaçu, Luiz Antônio, SP

Table 1 - Families, species, regional names, number of individuals $(N)$ and ecological group (GE) observed in a MogiGuaçu River floodplain, Luiz Antônio-SP, Brazil

\begin{tabular}{|c|c|c|c|c|}
\hline Famílias & Espécies & Nomes Regionais & $\mathrm{N}$ & $\mathrm{GE}^{1}$ \\
\hline Anacardiaceae & $\begin{array}{l}\text { Myracrodruon urundeuva Allemão } \\
\text { Schinus terebinthifolius Raddi }\end{array}$ & $\begin{array}{l}\text { Aroeira-verdadeira } \\
\text { Aroeira-mansa }\end{array}$ & $\begin{array}{c}13 \\
1\end{array}$ & $\begin{array}{l}\mathrm{C} \\
\mathrm{P}\end{array}$ \\
\hline Apocynaceae & $\begin{array}{l}\text { Aspidosperma polyneuron Muell. Arg. } \\
\text { Tabernaemontana hystrix (Steud.) A. DC. }\end{array}$ & $\begin{array}{l}\text { Peroba-rosa } \\
\text { Leiteiro }\end{array}$ & $\begin{array}{c}8 \\
74\end{array}$ & $\begin{array}{l}\mathrm{C} \\
\mathrm{P}\end{array}$ \\
\hline Arecaceae & Syagrus romanzoffiana (Cham.) Glassman & Jerivá & 3 & $\mathrm{~S}$ \\
\hline Bignoniaceae & $\begin{array}{l}\text { Tabebuia impetiginosa (Marte.) Standl. } \\
\text { Tabebuia roseo-alba (Ridley) Sand. }\end{array}$ & $\begin{array}{l}\text { Ipê-roxo } \\
\text { Ipê-branco }\end{array}$ & $\begin{array}{l}4 \\
1\end{array}$ & $\begin{array}{l}S \\
S\end{array}$ \\
\hline Cannabaceae & $\begin{array}{l}\text { Celtis iguanea (Jacq.) Sargent. } \\
\text { Trema micrantha (L.) Blume }\end{array}$ & $\begin{array}{l}\text { Joá-mirim, jameri } \\
\text { Candiúba }\end{array}$ & $\begin{array}{l}16 \\
33\end{array}$ & $\begin{array}{l}\mathrm{P} \\
\mathrm{P}\end{array}$ \\
\hline Clusiaceae & Calophyllum brasiliensis Cambess. & Guanandi & 14 & $\mathrm{C}$ \\
\hline \multirow[t]{3}{*}{ Euphorbiaceae } & Alchornea glandulosa Poepp \& Endl. & Tapiá, boleiro & 8 & $P$ \\
\hline & $\begin{array}{l}\text { Croton urucurana Baill. } \\
\text { Sebastiania commersoniana (Baill.) L. B. }\end{array}$ & $\begin{array}{l}\text { Sangra-d'água } \\
\text { Branquilho }\end{array}$ & $\begin{array}{l}34 \\
85\end{array}$ & $\begin{array}{l}\mathrm{P} \\
\mathrm{P}\end{array}$ \\
\hline & Mabea fistulifera Mart. & Canudo-de-pito & 2 & $\mathrm{P}$ \\
\hline \multirow[t]{4}{*}{ Fabaceae Caesalpinioideae } & $\begin{array}{l}\text { Cassia ferruginea (Schrad.) ex DC. } \\
\text { Copaifera langsdorffii Desf. }\end{array}$ & $\begin{array}{l}\text { Chuva-de-ouro } \\
\text { Óleo-de-copaíba }\end{array}$ & $\begin{array}{c}11 \\
9\end{array}$ & $\begin{array}{l}\mathrm{S} \\
\mathrm{S}\end{array}$ \\
\hline & Hymenaea courbaril L. var. stilbocarpa & Jatobá & 2 & $\mathrm{C}$ \\
\hline & Pterogyne nitens Tul. & Amendoim-bravo & 1 & $\mathrm{~S}$ \\
\hline & Schizolobium parahyba (Vell.) S.F. Blake & Guapuruvu & 1 & $\mathrm{~S}$ \\
\hline \multirow[t]{4}{*}{ Fabaceae Mimosoideae } & $\begin{array}{l}\text { Acacia polyphylla DC. } \\
\text { Albizia hasslerii (Chodat) Burr. }\end{array}$ & $\begin{array}{l}\text { Monjoleiro } \\
\text { Farinha-seca }\end{array}$ & $\begin{array}{c}169 \\
68\end{array}$ & $\begin{array}{l}\mathrm{P} \\
\mathrm{S}\end{array}$ \\
\hline & Anadenanthera macrocarpa (Benth.) Brenan & Angico-vermelho & 1 & $\mathrm{~S}$ \\
\hline & Enterolobium contortisiliquum (Vell.) Morong & Tamboril & 3 & $\mathrm{~S}$ \\
\hline & $\begin{array}{l}\text { Inga striata Benth. } \\
\text { Piptadenia gonoacantha (Mart.) J. F. Macbr. }\end{array}$ & $\begin{array}{l}\text { Ingá } \\
\text { Pau-jacaré }\end{array}$ & $\begin{array}{c}99 \\
5\end{array}$ & $\begin{array}{l}\mathrm{S} \\
\mathrm{P}\end{array}$ \\
\hline \multirow[t]{2}{*}{ Fabaceae Papilionoideae } & Machaerium stipitatum (DC.) Vogel & Sapuvinha & 2 & $\mathrm{~S}$ \\
\hline & Sweetia fruticosa (L.) Spreng. & Guaiçara & 1 & $\mathrm{C}$ \\
\hline Lauraceae & Nectandra megapotamica (Spreng.) Mez & Canelinha & 407 & $\mathrm{C}$ \\
\hline Magnoliaceae & Talauma ovata A. St.-Hil. & Pinha-do-brejo & 8 & $\mathrm{~S}$ \\
\hline Malvaceae & Apeiba tibourbou Aubl. & Pau-de-jangada & 62 & $\mathrm{~S}$ \\
\hline \multirow[t]{2}{*}{ Meliaceae } & Cabralea canjerana (Vell.) Mart. & Canjerana & 284 & $S$ \\
\hline & Guarea guidonia (L.) Sleumer & Marinheiro-do-brejo & 2 & $\mathrm{~S}$ \\
\hline Melastomataceae & Tibouchina mutabilis Cong. & Manacá-da-serra & 14 & $\mathrm{P}$ \\
\hline \multirow{2}{*}{ Myrsinaceae } & Rapanea ferruginea (Ruiz \& Pav.) Mez & Capororoca-vermelha & 38 & $\mathrm{P}$ \\
\hline & Rapanea guyanensis Aubl. & Capororoca-branca & 1 & $\mathrm{P}$ \\
\hline \multirow[t]{7}{*}{ Myrtaceae } & Eugenia florida D.C. & Pimenteira & 1 & $\mathrm{~S}$ \\
\hline & Eugenia pyriformis Cambess. & Uvaia & 3 & $\mathrm{~S}$ \\
\hline & Myrcia multiflora (Lam.) DC. & Cambuí & 1 & $\mathrm{~S}$ \\
\hline & Psidium cattleyanum Sabine & Araçá-do-campo & 229 & $\mathrm{~S}$ \\
\hline & Psidium guajava $\mathrm{L}$. & Goiaba & 39 & $\mathrm{~S}$ \\
\hline & Syzygium jambos (L.) Alston ${ }^{2}$ & Jambo & 1 & $\mathrm{Nc}$ \\
\hline & Syzygium cumini (L.) Skeels ${ }^{2}$ & Jambolão & 59 & $\mathrm{~S}$ \\
\hline Polygonaceae & Triplaris americana $\mathrm{L}$. & Pau-formiga & 20 & $\mathrm{~S}$ \\
\hline \multirow[t]{2}{*}{ Rubiaceae } & Genipa americana $\mathrm{L}$. & Jenipapo & 32 & $\mathrm{~S}$ \\
\hline & Guettarda viburnoides Cham. \& Schltdl. & Veludinho & 3 & $\mathrm{~S}$ \\
\hline \multirow[t]{2}{*}{ Rutaceae } & Esenbeckia grandiflora Mart. & Guaxupita, pau-de-cotia & 1 & $\mathrm{C}$ \\
\hline & Metrodorea nigra A. St. Hil. & Pau-fero & 1 & $\mathrm{~S}$ \\
\hline Salicaceae & Casearia sylvestris $\mathrm{Sw}$. & Guaçatonga & 10 & $\mathrm{~S}$ \\
\hline Sapindaceae & Sapindus saponaria $\mathrm{L}$. & Sabão-de-soldado & 2 & $\mathrm{~S}$ \\
\hline Urticaceae & Cecropia hololeuca Miq. & Embaúba & 93 & $\mathrm{P}$ \\
\hline Vochysiaceae & Qualea grandiflora Mart. & Pau-terra & 2 & $\mathrm{~S}$ \\
\hline \multirow[t]{2}{*}{ Indeterminadas } & Desconhecida 1 & - & 8 & $\mathrm{Nc}$ \\
\hline & Desconhecida 2 & - & 1 & $\mathrm{Nc}$ \\
\hline
\end{tabular}

${ }^{1} \mathrm{P}=$ pioneira, $\mathrm{S}=$ secundária, $\mathrm{C}$ = climácica e $\mathrm{Nc}=$ não classificada e ${ }^{2}$ espécie exótica.

R. Árvore, Viçosa-MG, v.32, n.5, p.909-920, 2008 
Em levantamento fitossociológico realizado nas mesmas áreas e analisando a vegetação arbórea (DAP $\geq 5,0 \mathrm{~cm}$ ), constatou-se a existência de 2.295 indivíduos distribuídos em 60 espécies arbóreas identificadas. Entre estas, 32 espécies foram encontradas no levantamento da regeneração natural. Quinze espécies da regeneração natural não apresentaram indivíduos na vegetação arbórea (indivíduos com DAP $\geq 5,0 \mathrm{~cm}$ ), sendo elas Aspidosperma polyneuron, Cabralea canjerana, Esenbeckia grandiflora, Eugenia pyriformis, Guarea guidonia, Guettarda viburnoides, Mabea fistulifera, Myracrodruon urundeuva, Myrcia multiflora, Pterogyne nitens, Qualea grandiflora, Rapanea guyanensis, Sapindus saponaria, Syzygium jambos e Talauma ovata. Esses resultados indicam que já estava ocorrendo regeneração de espécies no reflorestamento artificial e que essa prática favoreceu a presença de animais disseminadores de sementes, elementos indispensáveis ao processo de restauração de ecossistemas florestais.

O Índice de Similaridade (ISJ) encontrado entre a vegetação do estrato arbóreo (DAP $\geq 5 \mathrm{~cm}$ ) e a regeneração natural (DAP $<5 \mathrm{~cm}$ ) foi de $43 \%$. Essa similaridade entre as florestas plantadas e as nativas deverá aumentar ao longo do tempo, à medida que a sucessão avance, com base em Silva Júnior et al. (2004). Santiago et al. (2005), analisando a fitossociologia de árvores adultas antes de um incêndio em 1994 e dos indivíduos arbóreos de regeneração 6 anos após o incêndio, verificaram que $68 \%$ do total de 118 espécies em regeneração haviam sido encontradas no estrato arbóreo antes do incêndio. Antes do incêndio foram encontradas 99 espécies do estrato arbóreo. O incêndio causou a morte de cerca de $40 \%$ das árvores na mata, resultou em alterações no regime de luz no interior da mata e, conseqüentemente, favoreceu o estabelecimento de espécies intolerantes ao sombreamento.

Considerando os números de indivíduos, famílias, espécies e valores de diversidade de Shannon (H') nas seis áreas avaliadas, a diversidade de espécies foi maior nas áreas de reflorestamento com espécies nativas do que nos remanescentes naturais e no povoamento de eucalipto (Quadro 2). Os valores de eqüabilidade (J) indicam que a distribuição dos indivíduos por espécie foi mais regular e homogênea nas áreas de reflorestamento $\mathrm{R} 1$ e R3 em relação às demais áreas, onde ocorreu, relativamente, maior número de indivíduos de poucas espécies.

Esses valores estão próximos dos encontrados por Passos (1998) em Mata Ciliar do rio Mogi-Guaçu, Município de Mogi-Guaçu, SP, onde encontrou H' = 2,6 nats ind $^{-1}$ e $\mathrm{J}=0,8$. Entretanto, os valores de $\mathrm{H}^{\prime}$ das áreas deste estudo foram superiores ao encontrado por Durigan e Dias (1990) na Mata Ciliar da Fazenda Cananéia $\left(\mathrm{H}^{\prime}=1,39\right.$ nats ind $\left.{ }^{-1}\right)$.

Quadro 2 - Índice de diversidade de Shannon e Weaver (H') e eqüabilidade (J) das espécies arbóreas em regeneração (DAP $<5,0 \mathrm{~cm}$ ) nos remanescentes naturais (RIP1 e RIP2), em povoamento de Eucalyptus robusta e nas áreas reflorestadas (R1, R2 e R3) da várzea do rio Mogi-Guaçu, Luiz Antônio, SP

Table 2 - Shannon \& Weaver diversity index $\left(H^{\prime}\right)$ and equability $(J)$ of the arboreal species regeneration in the natural remainders (RIP1 e RIP2), in a Eucalyptus robusta plantation and in the reforestation areas with native species $(R 1, R 2$ e R3) at the Mogi-Guaçu River floodplain, Luiz, Antonio County, São Paulo State, Brazil

\begin{tabular}{|c|c|c|c|c|c|}
\hline \multirow[t]{2}{*}{ Áreas } & \multicolumn{3}{|c|}{ Quantidades (Números) } & \multicolumn{2}{|c|}{ Espécies } \\
\hline & Indivíduos & Famílias & Espécies & $\left(\mathrm{H}^{\prime}\right)$ & $(\mathrm{J})$ \\
\hline RIP1 & 270 & 13 & 14 & 1,9 & 0,7 \\
\hline RIP2 & 328 & 14 & 19 & 2,1 & 0,7 \\
\hline Eucalyptus & 243 & 14 & 21 & 1,9 & 0,6 \\
\hline Reflorestamento R1 & 416 & 16 & 22 & 2,5 & 0,8 \\
\hline Reflorestamento R2 & 448 & 18 & 33 & 2,4 & 0,7 \\
\hline \multirow[t]{3}{*}{ Reflorestamento R3 } & 285 & 14 & 23 & 2,5 & 0,8 \\
\hline & \multicolumn{5}{|c|}{ Grupos ecológicos das espécies (\%) } \\
\hline & Pioneiras & Secundárias & Climácicas & $\mathrm{Nc}^{1}$ & Total \\
\hline RIP1 & 25 & 70 & 5 & 0 & 100 \\
\hline RIP2 & 12 & 63 & 24 & 1 & 100 \\
\hline Eucalyptus & 16 & 32 & 51 & 1 & 100 \\
\hline Reflorestamento R1 & 34 & 38 & 27 & 1 & 100 \\
\hline Reflorestamento R2 & 51 & 31 & 17 & 1 & 100 \\
\hline Reflorestamento R3 & $39^{(2)}$ & 45 & 16 & 0 & 100 \\
\hline
\end{tabular}

${ }^{1} \mathrm{Nc}=$ não classificada e ${ }^{(2)}$ presença de $20 \%$ de Syzygium jambos, com comportamento de espécies pioneiras no reflorestamento. 
Verificou-se a predominância de espécies secundárias nos remanescentes florestais RIP1 e RIP2, sendo que as espécies com maiores valores de classe de tamanho $(\mathrm{Ct} \%)$, abundância relativa ( $\mathrm{Ab} \%)$, regeneração natural (Rn\%) e valor importância (VI) foram, respectivamente,
Cabralea canjerana e Psidium cattleyanum (Quadros 2 e 3). No povoamento de eucalipto, o sombreamento favoreceu a regeneração da espécie climácica Nectandra megapotamica, sendo a que apresentou os maiores valores de $\mathrm{Ct} \%, \mathrm{Ab} \%, \mathrm{Rn} \%$ e VI (Quadros 2 e 4).

Quadro 3 - Número de indivíduos presentes nas classes de altura Ct I, Ct II e CT III e parâmetros fitossociológicos das espécies arbóreas em regeneração nos remanescente naturais (RIP1 e RIP2) da várzea do rio Mogi-Guaçu, Luiz Antônio, SP

Table 3 -Number of individuals presented in Ct I, Ct II e CT III height classes and phytosociological parameters of arboreal species of the natural remainders (RIP1 and RIP2) in a Mogi-Guaçu River floodplain, Luiz Antônio-SP, Brazil

\begin{tabular}{|c|c|c|c|c|c|c|c|c|c|}
\hline \multirow[t]{2}{*}{ Espécies } & $\mathrm{Ct} \mathrm{I}$ & Ct II & $\mathrm{Ct}$ III & $\mathrm{Ct}$ IV & $\mathrm{Ct} \%$ & $\mathrm{Ab} \%$ & $\mathrm{Fr} \%$ & $\mathrm{Rn} \%$ & $\mathrm{VI}$ \\
\hline & \multicolumn{9}{|c|}{ RIP1 } \\
\hline Cabralea canjerana & 10 & 45 & 57 & - & 42,5 & 41,5 & 25,7 & 36,6 & 67,2 \\
\hline Psidium cattleyanum & 7 & 17 & 17 & - & 14,5 & 15,2 & 17,1 & 15,6 & 32,3 \\
\hline Tabernaemontana hystrix & 5 & 24 & 15 & - & 16,4 & 16,3 & 15,0 & 15,9 & 31,3 \\
\hline Sebastiania commersoniana & 1 & 8 & 11 & - & 7,8 & 7,4 & 10,0 & 8,4 & 17,4 \\
\hline Nectandra megapotamica & 1 & 5 & 8 & - & 5,4 & 5,2 & 7,9 & 6,1 & 13,0 \\
\hline Inga striata & 1 & 10 & 1 & - & 4,6 & 4,4 & 6,4 & 5,1 & 10,9 \\
\hline Albizia hasslerii & 4 & 3 & 0 & - & 1,6 & 2,6 & 5,0 & 3,1 & 7,6 \\
\hline Casearia sylvestris & 0 & 0 & 4 & - & 1,6 & 1,5 & 3,6 & 2,2 & 5,1 \\
\hline Apeiba tibourbou & 0 & 3 & 2 & - & 2,0 & 1,9 & 2,1 & 2,0 & 4,0 \\
\hline Celtis iguanea & 0 & 2 & 1 & - & 1,2 & 1,1 & 2,1 & 1,5 & 3,3 \\
\hline Genipa americana & 3 & 0 & 0 & - & 0,0 & 1,0 & 2,1 & 1,2 & 3,3 \\
\hline Syagrus romanzoffiana & 0 & 2 & 1 & - & 1,2 & 1,1 & 1,4 & 1,3 & 2,5 \\
\hline Machaerium stipitatum & 0 & 1 & 0 & - & 0,4 & 0,4 & 0,7 & 0,5 & 1,1 \\
\hline \multirow[t]{2}{*}{ Cecropia hololeuca } & 0 & 1 & 0 & - & 0,0 & 0,0 & 1,0 & 0,0 & 1,1 \\
\hline & \multicolumn{9}{|c|}{ RIP2 } \\
\hline Psidium cattleyanum & 20 & 42 & 17 & 4 & 27,0 & 25,3 & 22,3 & 24,9 & 47,6 \\
\hline Cabralea canjerana & 11 & 19 & 32 & 15 & 20,4 & 23,5 & 16,6 & 20,1 & 40,0 \\
\hline Nectandra megapotamica & 40 & 23 & 6 & 1 & 24,4 & 21,3 & 15,9 & 20,5 & 37,3 \\
\hline Genipa americana & 2 & 10 & 6 & 1 & 5,9 & 5,8 & 8,3 & 6,7 & 14,1 \\
\hline Inga striata & 3 & 7 & 5 & 2 & 5,1 & 5,2 & 8,3 & 6,2 & 13,5 \\
\hline Sebastiania commersoniana & 2 & 5 & 4 & 6 & 4,2 & 5,2 & 7,0 & 5,5 & 12,2 \\
\hline Albizia hasslerii & 5 & 1 & 1 & 0 & 2,4 & 2,1 & 4,5 & 3,0 & 6,6 \\
\hline Tabernaemontana hystrix & 1 & 1 & 3 & 1 & 1,6 & 1,8 & 2,6 & 2,0 & 4,4 \\
\hline Rapanea ferruginea & 2 & 0 & 2 & 1 & 1,3 & 1,5 & 2,6 & 1,8 & 4,1 \\
\hline Alchornea glandulosa & 2 & 0 & 1 & 2 & 1,2 & 1,5 & 2,6 & 1,8 & 4,1 \\
\hline Calophyllum brasiliense & 3 & 2 & 1 & 1 & 2,2 & 2,1 & 1,9 & 2,1 & 4,0 \\
\hline Croton urucurana & 2 & 2 & 0 & 0 & 1,5 & 1,2 & 2,6 & 1,7 & 3,8 \\
\hline Tibouchina mutabilis & 1 & 0 & 1 & 1 & 0,7 & 0,9 & 1,3 & 1,0 & 2,2 \\
\hline Cassia ferruginea & 0 & 2 & 0 & 1 & 0,8 & 0,9 & 0,6 & 0,8 & 1,6 \\
\hline Desconhecida 1 & 1 & 0 & 0 & 0 & 0,4 & 0,3 & 0,6 & 0,4 & 0,9 \\
\hline Machaerium stipitatum & 1 & 0 & 0 & 0 & 0,4 & 0,3 & 0,6 & 0,4 & 0,9 \\
\hline Desconhecida 2 & 0 & 0 & 1 & 0 & 0,2 & 0,3 & 0,6 & 0,4 & 0,9 \\
\hline Syzygium Jambos & 0 & 0 & 1 & 0 & 0,2 & 0,3 & 0,6 & 0,4 & 0,9 \\
\hline Eugenia florida & 0 & 0 & 0 & 1 & 0,1 & 0,3 & 0,6 & 0,4 & 0,9 \\
\hline
\end{tabular}

$\mathrm{Ct} \%$ = categoria de tamanho relativa, $\mathrm{Ab} \%$ = abundância relativa, $\mathrm{Fr} \%$ = frequiência relativa, $\mathrm{Rn} \%$ = regeneração natural relativa e $\mathrm{VI}=$ valor de importância.

R. Árvore, Viçosa-MG, v.32, n.5, p.909-920, 2008 
Quadro 4 - Número de indivíduos presentes nas classes de tamanho Ct I, Ct II, CT III e Ct IV e parâmetros fitossociológicos das espécies arbóreas em regeneração no povoamento de Eucalyptus robusta (E) e no reflorestamento com espécies nativas (R1) na várzea do rio Mogi-Guaçu, Luiz Antônio, SP

Table 4-Number of individuals presented in Ct I, Ct II e CT III height classes and phytosociological parameters of arboreal species of the Eucalyptus robusta plantation (E) and reforestation area (R1) in a Mogi-Guaçu River floodplain, Luiz, Antônio-SP, Brazil

\begin{tabular}{|c|c|c|c|c|c|c|c|c|c|}
\hline \multirow[t]{2}{*}{ Espécies } & $\mathrm{Ct} \mathrm{I}$ & $\mathrm{Ct}$ II & $\mathrm{Ct}$ III & Ct IV & $\mathrm{Ct} \%$ & $\mathrm{Ab} \%$ & Fr\% & $\mathrm{Rn} \%$ & VI \\
\hline & \multicolumn{9}{|c|}{$\mathrm{E}$} \\
\hline Nectandra megapotamica & 12 & 34 & 26 & 43 & 348,2 & 47,3 & 28,5 & 39,3 & 75,8 \\
\hline Cabralea canjerana & 4 & 2 & 3 & 17 & 97,9 & 10,7 & 13,8 & 12,1 & 24,5 \\
\hline Psidium cattleyanum & 0 & 2 & 10 & 7 & 61,1 & 7,8 & 12,3 & 9,2 & 20,1 \\
\hline Apeiba tibourbou & 1 & 3 & 5 & 12 & 77,3 & 8,6 & 10,0 & 9,3 & 18,6 \\
\hline Cecropia hololeuca & 0 & 0 & 0 & 16 & 79,0 & 6,6 & 7,7 & 7,9 & 14,3 \\
\hline Rapanea ferruginea & 0 & 3 & 6 & 7 & 54,0 & 6,6 & 7,7 & 6,9 & 14,3 \\
\hline Calophyllum brasiliense & 0 & 0 & 1 & 7 & 31,8 & 2,9 & 5,4 & 4,0 & 6,3 \\
\hline Guettarda viburnoides & 0 & 0 & 0 & 3 & 14,8 & 1,2 & 1,5 & 1,5 & 2,8 \\
\hline Desconhecida 1 & 0 & 0 & 0 & 2 & 9,8 & 0,8 & 1,5 & 1,1 & 2,3 \\
\hline Acacia polyphylla & 0 & 0 & 1 & 1 & 7,2 & 0,8 & 1,5 & 1,0 & 2,3 \\
\hline Guarea guidonia & 0 & 0 & 1 & 1 & 7,2 & 0,8 & 1,5 & 1,0 & 2,3 \\
\hline Albizia hasslerii & 2 & 0 & 0 & 0 & 1,6 & 0,8 & 1,5 & 0,8 & 2,3 \\
\hline Genipa americana & 0 & 0 & 0 & 2 & 9,8 & 0,8 & 0,7 & 0,9 & 1,6 \\
\hline Tibouchina mutabilis & 0 & 0 & 0 & 2 & 9,8 & 0,8 & 0,7 & 0,9 & 1,6 \\
\hline Qualea grandiflora & 0 & 1 & 1 & 0 & 4,2 & 0,8 & 0,7 & 0,7 & 1,6 \\
\hline Metrodorea nigra & 0 & 0 & 0 & 1 & 4,9 & 0,4 & 0,7 & 0,6 & 1,2 \\
\hline Sweetia fruticosa & 0 & 0 & 1 & 0 & 2,2 & 0,4 & 0,7 & 0,5 & 1,2 \\
\hline Inga striata & 0 & 1 & 0 & 0 & 1,9 & 0,4 & 0,7 & 0,5 & 1,2 \\
\hline Rapanea guyanensis & 0 & 1 & 0 & 0 & 1,9 & 0,4 & 0,7 & 0,5 & 1,2 \\
\hline Trema micrantha & 0 & 1 & 0 & 0 & 1,9 & 0,4 & 0,7 & 0,5 & 1,2 \\
\hline \multirow[t]{2}{*}{ Esenbeckia grandiflora } & 1 & 0 & 0 & 0 & 0,8 & 0,4 & 0,7 & 0,4 & 1,2 \\
\hline & \multicolumn{9}{|c|}{$\mathrm{R} 1$} \\
\hline Nectandra megapotamica & 29 & 18 & 21 & 43 & 24,3 & 26,7 & 12,7 & 21,2 & 39,4 \\
\hline Inga striata & 6 & 5 & 14 & 9 & 7,2 & 8,2 & 11,9 & 9,1 & 20,1 \\
\hline Cecropia hololeuca & 1 & 0 & 16 & 22 & 11,0 & 9,4 & 7,1 & 9,2 & 16,5 \\
\hline Cabralea canjerana & 5 & 7 & 9 & 13 & 7,6 & 8,2 & 7,1 & 7,7 & 15,3 \\
\hline Sebastiania commersoniana & 2 & 2 & 8 & 31 & 12,7 & 10,3 & 4,8 & 9,3 & 15,1 \\
\hline Albizia hasslerii & 0 & 8 & 17 & 13 & 8,8 & 9,1 & 5,6 & 7,8 & 14,7 \\
\hline Apeiba tibourbou & 1 & 2 & 5 & 11 & 5,1 & 4,6 & 6,4 & 5,4 & 10,9 \\
\hline Triplaris americana & 8 & 3 & 0 & 0 & 1,2 & 2,6 & 6,4 & 3,4 & 9,0 \\
\hline Trema micrantha & 0 & 0 & 3 & 16 & 6,1 & 4,6 & 3,2 & 4,6 & 7,7 \\
\hline Psidium cattleyanum & 4 & 4 & 3 & 1 & 1,8 & 2,9 & 4,8 & 3,2 & 7,7 \\
\hline Celtis iguanea & 0 & 0 & 6 & 4 & 2,6 & 2,4 & 4,8 & 3,3 & 7,2 \\
\hline Tabernaemontana hystrix & 0 & 1 & 4 & 8 & 3,7 & 3,1 & 4,0 & 3,6 & 7,1 \\
\hline Cassia ferruginea & 3 & 2 & 0 & 1 & 0,9 & 1,4 & 4,0 & 2,1 & 5,4 \\
\hline Genipa americana & 2 & 1 & 1 & 1 & 0,9 & 1,2 & 4,0 & 2,0 & 5,2 \\
\hline Croton urucurana & 1 & 0 & 2 & 4 & 1,9 & 1,7 & 3,2 & 2,3 & 4,9 \\
\hline Rapanea ferruginea & 0 & 0 & 0 & 5 & 1,7 & 1,2 & 3,2 & 2,0 & 4,4 \\
\hline Desconhecida 1 & 1 & 0 & 2 & 1 & 0,9 & 1,0 & 1,6 & 1,1 & 2,6 \\
\hline Piptadenia gonoacantha & 0 & 0 & 0 & 2 & 0,7 & 0,5 & 1,6 & 0,9 & 2,1 \\
\hline Schinus terebinthifolius & 0 & 0 & 0 & 1 & 0,3 & 0,2 & 1,6 & 0,7 & 1,8 \\
\hline Tibouchina mutabilis & 0 & 0 & 1 & 0 & 0,2 & 0,2 & 0,8 & 0,4 & 1,0 \\
\hline Copaifera langsdorffii & 0 & 1 & 0 & 0 & 0,1 & 0,2 & 0,8 & 0,4 & 1,0 \\
\hline Psidium guajava & 0 & 1 & 0 & 0 & 0,1 & 0,2 & 0,8 & 0,4 & 1,0 \\
\hline
\end{tabular}

$\mathrm{Ct} \%=$ categoria de tamanho relativa, $\mathrm{Ab} \%=$ abundância relativa, Fr\% = freqüência relativa, $\mathrm{Rn} \%=$ regeneração natural relativa e $\mathrm{VI}=$ valor de importância. 
No reflorestamento mais antigo (R1), por apresentar um dossel mais fechado do que os outros dois estudados, também houve regeneração considerável de N. megapotamica (Quadros 2 e 4). Nos reflorestamentos mais jovens (R2 e R3), houve predominância de espécies pioneiras e secundárias, respectivamente (Quadros 2, 5 e 6). Nos reflorestamentos R2 e R3, as espécies mais abundantes, com maiores índices de regeneração natural e valores de importância, foram, respectivamente, a pioneira Acacia polyphylla e a exótica Syzygium cumini (jambolão). Esta última apresenta comportamento de espécie pioneira e tende a formar povoamentos homogêneos. Como medida de manejo, recomenda-se a sua erradicação do local. A alta porcentagem de espécies pioneiras nessa fase de regeneração é reflexo de a área ainda apresentar o dossel aberto. Vários trechos das áreas reflorestadas apresentaram grande densidade de gramíneas, o que dificulta a regeneração natural. Os resultados indicaram que, no período decorrido entre o plantio e o fechamento do dossel nas áreas reflorestadas, houve regime de luz que favoreceu o estabelecimento de espécies pioneiras, ou seja, intolerantes, como observaram Santiago et al. (2005) em área de Cerrado após incêndio.

Quadro 5 - Número de indivíduos presentes nas classes de tamanho Ct I, Ct II, CT III e Ct IV e parâmetros fitossociológicos das espécies arbóreas em regeneração na área de reflorestamento (R2) na várzea do rio Mogi-Guaçu, Luiz Antônio, SP

Table 5 -Number of individuals presented in Ct I, Ct II e CT III height classes and phytosociological parameters of arboreal species of the reforestation area (R2) in a Mogi-Guaçu River floodplain, Luiz,Antônio-SP, Brazil

\begin{tabular}{|c|c|c|c|c|c|c|c|c|c|}
\hline Espécies & $\mathrm{Ct} \mathrm{I}$ & $\mathrm{Ct}$ II & $\mathrm{Ct} \mathrm{III}$ & $\mathrm{Ct}$ IV & $\mathrm{Ct} \%$ & $\mathrm{Ab} \%$ & $\mathrm{Fr} \%$ & $\mathrm{Rn} \%$ & VI \\
\hline$\overline{\text { Acacia polyphylla }}$ & 101 & 50 & 14 & 2 & 44,5 & 37,3 & 5,5 & 29,1 & 42,7 \\
\hline Nectandra megapotamica & 17 & 13 & 26 & 8 & 12,8 & 14,3 & 12,5 & 13,2 & 26,8 \\
\hline Psidium cattleyanum & 21 & 3 & 3 & 4 & 7,6 & 6,9 & 8,6 & 7,7 & 15,5 \\
\hline Cabralea canjerana & 10 & 11 & 6 & 2 & 6,8 & 6,5 & 8,6 & 7,3 & 15,1 \\
\hline Cecropia hololeuca & 2 & 2 & 15 & 12 & 4,4 & 6,9 & 7,0 & 6,1 & 14,0 \\
\hline Apeiba tibourbou & 5 & 3 & 6 & 3 & 3,3 & 3,8 & 7,0 & 4,7 & 10,8 \\
\hline Trema micrantha & 3 & 1 & 2 & 7 & 2,0 & 2,9 & 4,7 & 3,2 & 7,6 \\
\hline Inga striata & 3 & 4 & 4 & 2 & 2,8 & 2,9 & 3,9 & 3,2 & 6,8 \\
\hline Rapanea ferruginea & 0 & 1 & 4 & 3 & 1,1 & 1,8 & 3,1 & 2,0 & 4,9 \\
\hline Copaifera langsdorffii & 1 & 0 & 0 & 5 & 1,0 & 1,3 & 3,1 & 1,8 & 4,5 \\
\hline Aspidosperma polyneuron & 4 & 0 & 1 & 3 & 1,6 & 1,8 & 2,3 & 1,9 & 4,1 \\
\hline Psidium guajava & 1 & 1 & 1 & 1 & 0,8 & 0,9 & 3,1 & 1,6 & 4,0 \\
\hline Croton urucurana & 1 & 0 & 1 & 2 & 0,5 & 0,9 & 3,1 & 1,5 & 4,0 \\
\hline Albizia hasslerii & 3 & 0 & 1 & 1 & 1,1 & 1,1 & 2,3 & 1,5 & 3,5 \\
\hline Casearia sylvestris & 3 & 0 & 2 & 0 & 1,1 & 1,1 & 2,3 & 1,5 & 3,5 \\
\hline Eugenia pyriformis & 3 & 0 & 0 & 0 & 0,9 & 0,7 & 2,3 & 1,3 & 3,0 \\
\hline Enterolobium contortisiliquит & 0 & 0 & 1 & 2 & 0,3 & 0,7 & 2,3 & 1,1 & 3,0 \\
\hline Genipa americana & 0 & 0 & 0 & 3 & 0,3 & 0,7 & 2,3 & 1,1 & 3,0 \\
\hline Talauma ovata & 5 & 2 & 1 & 0 & 2,2 & 1,8 & 0,8 & 1,6 & 2,6 \\
\hline Piptadenia gonoacantha & 0 & 0 & 0 & 3 & 0,1 & 0,7 & 1,6 & 0,8 & 2,2 \\
\hline Myracrodruon urundeuva & 1 & 1 & 0 & 0 & 0,7 & 0,4 & 1,6 & 0,9 & 2,0 \\
\hline Sapindus saponaria & 1 & 1 & 0 & 0 & 0,7 & 0,4 & 1,6 & 0,9 & 2,0 \\
\hline Triplaris americana & 1 & 1 & 0 & 0 & 0,7 & 0,4 & 1,6 & 0,9 & 2,0 \\
\hline Mabea fistulifera & 0 & 1 & 0 & 1 & 0,4 & 0,4 & 1,6 & 0,8 & 2,0 \\
\hline Tabebuia impetiginosa & 0 & 0 & 1 & 3 & 0,4 & 0,9 & 0,8 & 0,7 & 1,7 \\
\hline Syzygium cumini & 2 & 1 & 0 & 0 & 0,9 & 0,7 & 0,8 & 0,8 & 1,5 \\
\hline Sebastiania commersoniana & 0 & 0 & 0 & 2 & 0,3 & 0,4 & 0,8 & 0,5 & 1,2 \\
\hline Cassia ferruginea & 0 & 0 & 1 & 0 & 0,2 & 0,2 & 0,8 & 0,4 & 1,0 \\
\hline Desconhecida & 0 & 1 & 0 & 0 & 0,2 & 0,2 & 0,8 & 0,4 & 1,0 \\
\hline Myrcia multiflora & 0 & 0 & 1 & 0 & 0,2 & 0,2 & 0,8 & 0,4 & 1,0 \\
\hline Pterogyne nitens & 0 & 0 & 0 & 1 & 0,2 & 0,2 & 0,8 & 0,4 & 1,0 \\
\hline Tabebuia roseo-alba & 0 & 0 & 0 & 1 & 0,2 & 0,2 & 0,8 & 0,4 & 1,0 \\
\hline Tabernaemontana hystrix & 1 & 0 & 0 & 0 & 0,2 & 0,2 & 0,8 & 0,4 & 1,0 \\
\hline
\end{tabular}

$\mathrm{Ct} \%=$ categoria de tamanho relativa, $\mathrm{Ab} \%=$ abundância relativa, Fr\% = freqüência relativa, $\mathrm{Rn} \%=$ regeneração natural relativa e $\mathrm{VI}=$ valor de importância.

R. Árvore, Viçosa-MG, v.32, n.5, p.909-920, 2008 
Quadro 6 - Número de indivíduos presentes nas classes de tamanho Ct I, Ct II, CT III e Ct IV e parâmetros fitossociológicos das espécies arbóreas em regeneração na área de reflorestamento (R3) na várzea do rio Mogi-Guaçu, Fazenda Guatapará da Votorantim Celulose e Papel, Luiz Antônio, SP

Table 6-Number of individuals presented in Ct I, Ct II e CT III height classes and phytosociological parameters of arboreal species of the reforestation area (R3) in a Mogi-Guaçu River floodplain, Luiz, Antônio-SP, Brazil

\begin{tabular}{|c|c|c|c|c|c|c|c|c|c|}
\hline Espécies & $\mathrm{Ct} \mathrm{I}$ & $\mathrm{Ct}$ II & Ct III & $\mathrm{Ct}$ IV & $\mathrm{Ct} \%$ & $\mathrm{Ab} \%$ & $\mathrm{Fr} \%$ & $\mathrm{Rn} \%$ & VI \\
\hline Syzygium cumini & 33 & 12 & 9 & 2 & 18,8 & 18,9 & 8,8 & 15,5 & 27,6 \\
\hline Psidium cattleyanum & 9 & 6 & 13 & 15 & 14,6 & 14,5 & 12,3 & 13,8 & 26,8 \\
\hline Nectandra megapotamica & 13 & 11 & 15 & 6 & 15,1 & 15,2 & 11,4 & 13,9 & 26,7 \\
\hline Psidium guajava & 12 & 10 & 9 & 5 & 11,4 & 11,4 & 10,5 & 11,1 & 22,0 \\
\hline Inga striata & 1 & 1 & 6 & 14 & 7,4 & 7,4 & 5,3 & 6,7 & 12,7 \\
\hline Croton urucurana & 0 & 0 & 1 & 18 & 6,4 & 6,4 & 6,1 & 6,3 & 12,5 \\
\hline Myracrodruon urundeuva & 0 & 0 & 2 & 9 & 3,7 & 3,7 & 6,1 & 4,5 & 9,8 \\
\hline Tabernaemontana hystrix & 1 & 2 & 2 & 5 & 3,3 & 3,4 & 5,3 & 4,0 & 8,6 \\
\hline Tibouchina mutabilis & 3 & 2 & 1 & 2 & 2,8 & 2,7 & 4,4 & 3,3 & 7,1 \\
\hline Triplaris americana & 1 & 1 & 3 & 2 & 2,2 & 2,4 & 4,4 & 3,0 & 6,7 \\
\hline Albizia hasslerii & 0 & 0 & 3 & 6 & 3,1 & 3,0 & 3,5 & 3,2 & 6,5 \\
\hline Cabralea canjerana & 5 & 1 & 0 & 0 & 2,0 & 2,0 & 2,6 & 2,2 & 4,7 \\
\hline Cecropia hololeuca & 0 & 0 & 2 & 4 & 2,0 & 2,0 & 2,6 & 2,2 & 4,7 \\
\hline Rapanea ferruginea & 0 & 1 & 0 & 3 & 1,4 & 1,4 & 2,6 & 1,8 & 4,0 \\
\hline Alchornea glandulosa & 0 & 0 & 1 & 2 & 1,2 & 1,0 & 2,6 & 1,6 & 3,6 \\
\hline Celtis iguanea & 0 & 1 & 2 & 0 & 1,2 & 1,0 & 2,6 & 1,6 & 3,6 \\
\hline Sebastiania commersoniana & 1 & 2 & 0 & 0 & 1,2 & 1,0 & 2,6 & 1,6 & 3,6 \\
\hline Hymenaea courbaril & 0 & 1 & 0 & 1 & 0,5 & 0,7 & 1,8 & 1,0 & 2,4 \\
\hline Copaifera langsdorffii & 1 & 0 & 1 & 0 & 0,5 & 0,7 & 0,9 & 0,7 & 1,6 \\
\hline Anadenanthera macrocarpa & 0 & 0 & 0 & 1 & 0,3 & 0,3 & 0,9 & 0,5 & 1,2 \\
\hline Casearia sylvestris & 0 & 0 & 1 & 0 & 0,3 & 0,3 & 0,9 & 0,5 & 1,2 \\
\hline Cassia ferruginea & 0 & 0 & 0 & 1 & 0,3 & 0,3 & 0,9 & 0,5 & 1,2 \\
\hline Schizolobium parahyba & 0 & 0 & 0 & 1 & 0,3 & 0,3 & 0,9 & 0,5 & 1,2 \\
\hline
\end{tabular}

$\mathrm{Ct} \%=$ categoria de tamanho relativa, $\mathrm{Ab} \%=$ abundância relativa, Fr\% = freqüência relativa, $\mathrm{Rn} \%=$ regeneração natural relativa e VI $=$ valor de importância.

A diversidade de espécies foi um pouco maior no remanescente de maior tamanho e com formato mais circular (RIP2) do que no RIP1, faixa ciliar de aproximadamente $100 \mathrm{~m}$ de largura às margens do rio Mogi-Guaçu (Quadro 2). O formato do povoamento, a umidade do solo e o efeito de borda influenciaram na distribuição das espécies na paisagem. Oliveira e Felfili (2005), estudando a estrutura e diâmetro da regeneração natural de uma mata de galeria no Distrito Federal, detectaram associação das categorias de regeneração com os ambientes, assim como espécies preferenciais tanto de ambientes de borda quanto de interior de mata.

Silva et al. (2003) consideraram que um trecho de floresta submontana da Fazenda São Geraldo, Viçosa, MG, encontrava-se em estádio médio de sucessão secundária e em franco desenvolvimento na fase madura, pois foram constatados cerca de $63 \%$ de espécies secundárias iniciais, $27 \%$ de secundárias tardias e $10 \%$ de pioneiras.
Ressalta-se que, no povoamento de Eucalyptus robusta, onúmero de espécies foi superior ao das áreas de remanescentes naturais RIP1 e RIP2 (Quadro 1), porém inferior ao encontrado por Durigan (1997), que amostrou 49 espécies num talhão de Eucalyptus citriodora e 38 espécies no Cerradão na Estação Experimental de Assis, SP.

No povoamento de eucalipto, a diversidade de espécies foi semelhante ao da área RIP1 $\left(\mathrm{H}^{\prime}=1,9\right.$ nats ind $\left.{ }^{-1}\right)$, porém superior ao valor de 0,7 nats ind ${ }^{-1}$ encontrado por Rezende (1995) na regeneração natural de sub-bosque de Eucalyptus grandis, no Município de Viçosa, MG. Tabarelli et al. (1993), comparando a vegetação do sub-bosque de um povoamento de Eucalyptus sp, localizado na Serra do Mar, SP, com uma vegetação secundária inicial próxima à floresta implantada e com a mesma idade demonstraram que, em algumas áreas, o sub-bosque de Eucalyptus sp possui maior riqueza de espécies e grupos ecológicos. Os referidos autores atribuíram essa riqueza a uma alta heterogeneidade interna no povoamento que proporciona a existência de vários nichos ecológicos.

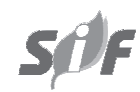

R. Árvore, Viçosa-MG, v.32, n.5, p.909-920, 2008 
Calegario et al. (1993), analisando a regeneração natural no Município de Belo Oriente, MG, em povoamentos de Eucalyptus paniculata e Eucalyptus grandis, identificaram 56 espécies nativas de 33 famílias botânicas distribuídas
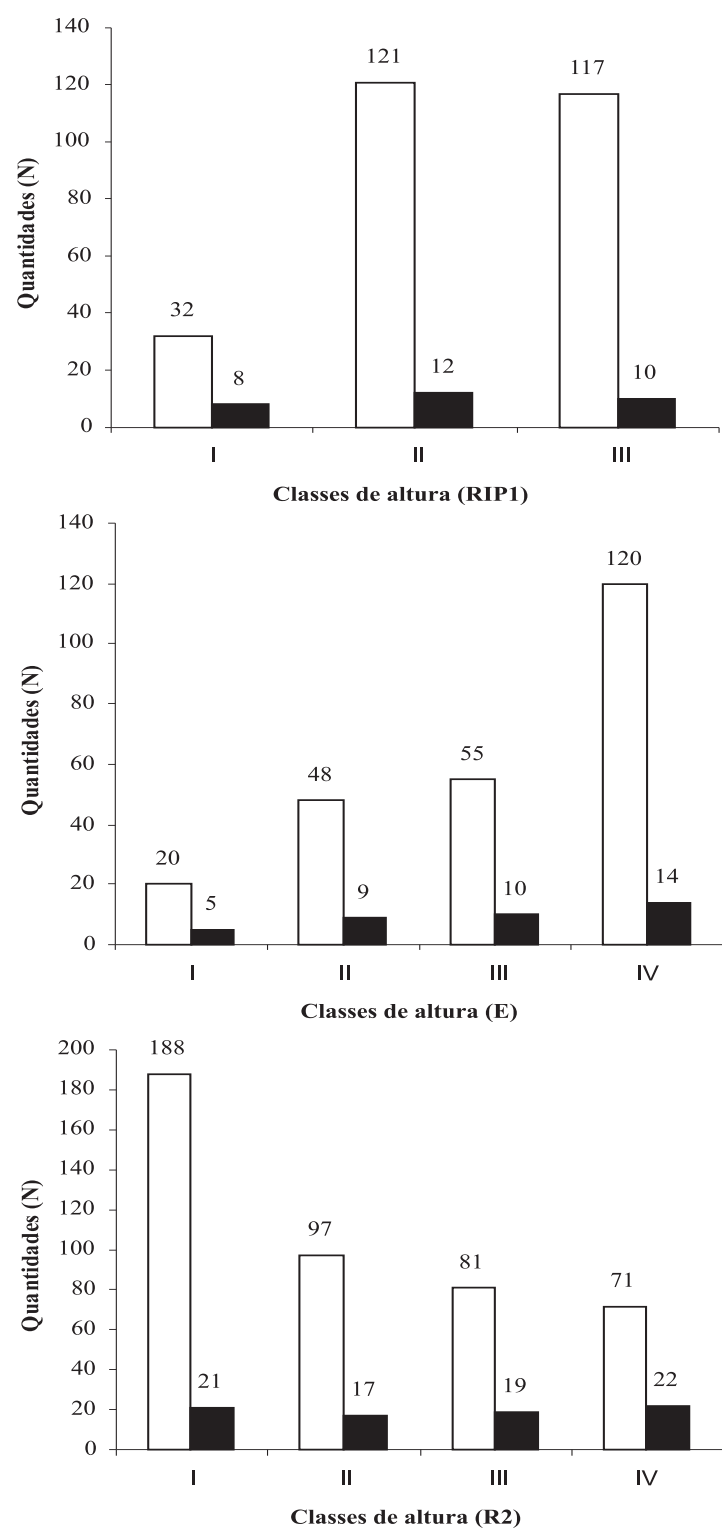

$\square$ Número de indivíduos nos dois povoamentos, destacando o povoamento de Eucalyptus grandis, por apresentar maior riqueza florística, devido à maior penetração de luz, facilitando a regeneração de espécies nativas.
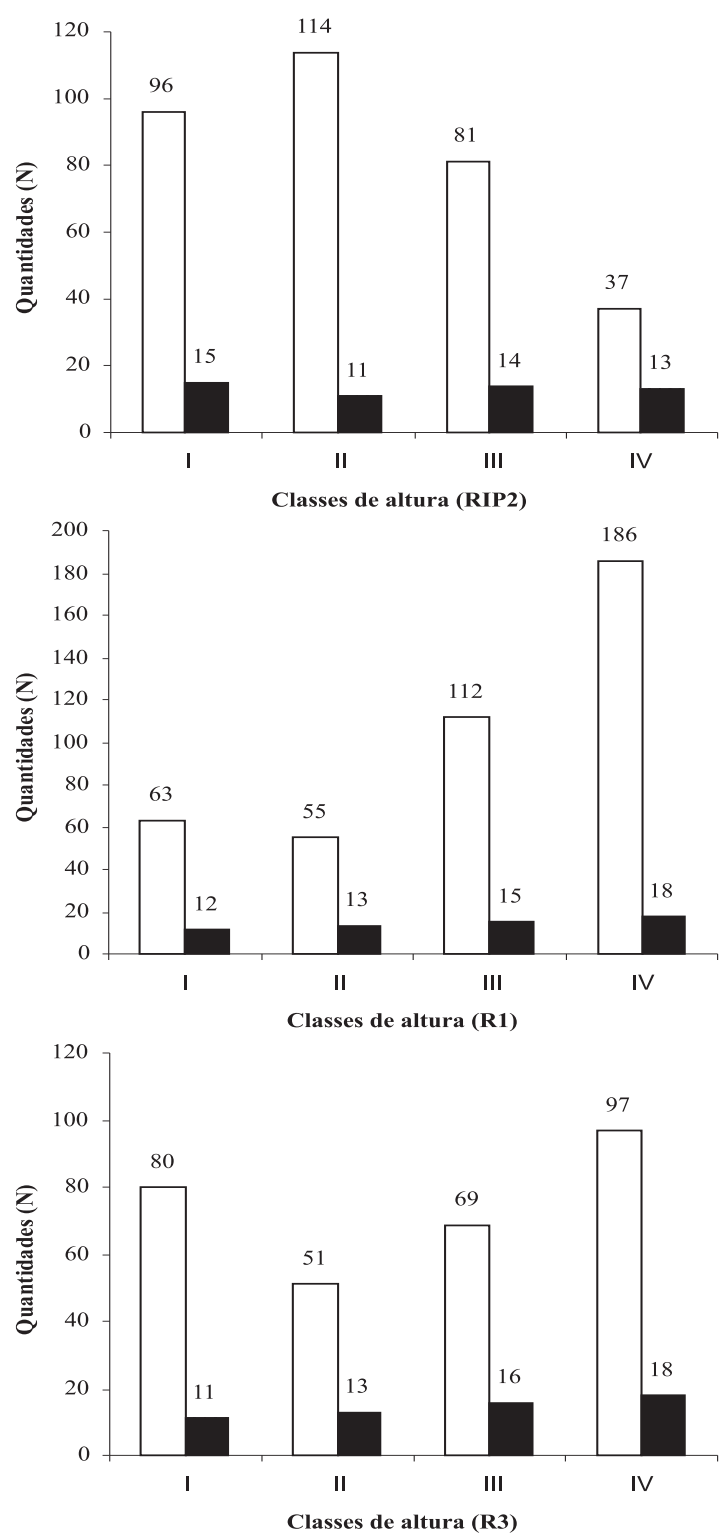

Número de espécies

Figura 1 - Número (N) de indivíduos e de espécies por classes de altura, I (0,10 - 0,50 m), II (0,51 - 1,0 m), III (1,0 $2,0 \mathrm{~m})$ e IV $(>2,0 \mathrm{~m}$ e DAP $\leq 5,0 \mathrm{~cm}$ ) dos remanescentes naturais (RIP1 e RIP2), povoamento de eucalipto (E) e áreas reflorestadas (R1, R2 e R3) da várzea do rio Mogi-Guaçu, Luiz Antônio, SP.

Figure 1 - Number $(N)$ of individuals and species per height class, I $(0,10-0,50 \mathrm{~m}), I I(0,51-1,0 \mathrm{~m}), I I I(1,0-2,0$ $m)$ e $I V(>2,0 \mathrm{~m}$ e DAP $\leq$ a 5,0 cm) of the natural remainders (RIP1 and RIP2), Eucalyptus robusta plantation (E) and of reforestation areas (R1, R2 and R3) in a Mogi-Guaçu River floodplain, Luiz Antoni-SP, Brazil.

R. Árvore, Viçosa-MG, v.32, n.5, p.909-920, 2008 
Com base nos dados de classes de tamanho (Quadros 2 a 6), as espécies Cabralea canjerana, Psidium cattleyanum, Nectandra megapotamica, Acacia polyphylla e Syzygium cumini estão entre as espécies representadas nas quatro categorias de tamanho, apresentando uma sequiência mais regular nas fases de crescimento e, portanto, maiores possibilidades de sobrevivência na estrutura futura da floresta do local.

A distribuição do número de indivíduos e de espécies nas diferentes classes de altura da regeneração variaram entre os remanescentes naturais e com o tipo, idade e local do reflorestamento (Figura 1). No subbosque do povoamento de eucalipto, destacaram-se o maior número e indivíduos e de espécies da maior classe de altura (IV), semelhante ao que ocorreu no reflorestamento mais antigo (R1). Nos remanescentes naturais RIP1 e RIP2, a distribuição dos indivíduos e espécies foi maior nas classes de altura intermediárias, entre 0,51 e $2 \mathrm{~m}$ (Ct II e Ct III).

\section{CONCLUSÕES}

O reflorestamento com espécies nativas em áreas degradadas da várzea do rio Mogi-Guaçu promoveu a regeneração natural com biodiversidade superior aos remanescentes naturais de florestas ciliares sob efeito de borda e contribuiu com o processo de restauração de ecossistemas florestais.

O povoamento de Eucalyptus robusta com cerca de 20 anos de idade favoreceu a regeneração de espécies climácicas e secundárias.

\section{AGRADECIMENTOS}

Os autores agradecem à Votorantim Celulose e Papel, pela acolhida e oportunidade para a realização deste trabalho, disponibilizando a área experimental, recursos humanos e financeiros; e ao Prof. Dr. Vinícius Castro Souza, curador do Herbário do Departamento de Botânica da Escola Superior de Agricultura "Luiz de Queiroz," da Universidade de São Paulo, pelos auxílios prestados na identificação das espécies.

\section{REFERÊNCIAS}

BARBOSA, L. M. Considerações gerais e modelos de recuperação de formações ciliares. In: RODRIGUES, R. R.; LEITÃO-FILHO, H. F. Matas ciliares: conservação e recuperação. São Paulo: Edusp/Fapesp, 2000. p.289-312.
BARBOSA, L. M. (Coord.). Manual para recuperação de áreas degradadas do estado de São Paulo: matas ciliares do interrior paulista. São Paulo: Instituto de Botânica, 2006. 128p.

BUDOWSKY, G. Distribution of tropical american rain forest species in the Light of cucessional processes. Turrialba, v.15, n.1, p.40-42, 1965.

CALDATO, S. L. et al. Estudo da regeneração natural, banco de sementes e Chuva de sementes na reserva genética Florestal de caçador, SC. Ciência Florestal, v.6, n.1, p.27-38. 1996.

CALEGARIO, N. et al. Parâmetros florísticos e fitossociológicos da regeneração natural de espécies arbóreas nativas no sub-bosque de povoamento de Eucaliptos. Revista Árvore, v.17, n.1, p.16-29, 1993.

CARVAlho, J. O. P. Manejo de regeneração natural de espécies florestais. Belém: Embrapa, 1984. 22p.

CARVALHO, P. E. R. Espécies arbóreas brasileiras. Brasília: Embrapa Informação Tecnológica; Colombo: Embrapa florestas. 2003. 1039p.

DANIEL, O.; JANKAUSKIS, J. Avaliação de metodologia para o estudo do estoque de sementes do solo. Série IPEF, v.41-42, p.18-26, 1989.

DURIGAN, G. Regeneração natural da vegetação de cerrado sob floresta de Eucalyptus citriodora. Revista do Instituto Florestal, v.9, n.1, p.71-85, 1997.

DURIGAN, G.; DIAS, H. C. S. Abundância da regeneração natural sob mata ciliar implantada. In. CONGRESSO FLORESTAL BRASILEIRO, 6., 1990, Campos do Jordão. Anais... Campos do Jordão: SBS, 1990. p.308-311.

\section{EMPRESA BRASILEIRA DE PESQUISA} AGROPECUÁRIA - EMBRAPA. Sistema brasileiro de classificação de solos. Rio de Janeiro: 1999. 412p.

FARIA, H. H.; SÉRGIO, F. C.; GARRIDO, M. A. O. Recomposição da vegetação ciliar integrada à conservação de microbacia. Revista do Instituto Florestal, n.21, p.1-22, 2001.

FINOL, U. H. Nuevos parámetros a considerarse en el análisis estructural de las selvas vírgenes tropicales. Revista Forestal Venezoelana, v.14, n.21, p.29-42, 1971.

R. Árvore, Viçosa-MG, v.32, n.5, p.909-920, 2008 
FISZON, J. T. et al. Causas antrópicas. In: RAMBALDI, D. M.; OLIVEIRA, D. A.S. (Orgs.) Fragmentação de ecossistemas: causas, efeitos sobre a biodiversidade e recomendações de políticas públicas. Brasília: MMA/SBF, 2003. p.65-99.

KRONKA, F. J. N. et al. Monitoramento da vegetação natural e do reflorestamento no Estado de São Paulo. In: SIMPÓSIO BRASILEIRO DE SENSORIAMENTO REMOTO, 12., 2005, Goiânia. Anais... São José dos Campos: Instituto Nacional de Pesquisas Espaciais, 2005. p.1569-1576.

LEITÃO-FILHO, H. F. Aspectos taxonômicos das florestas do Estado de São Paulo. Silvicultura em São Paulo, v.1, n.16, p.197-206, 1982.

LORENZI, H. Árvores brasileiras: manual de identificação de plantas arbóreas nativas do Brasil. Nova Odessa: Plantarum, 1992. 352p.

LORENZI, H. Árvores brasileiras: manual de identificação de plantas arbóreas nativas do Brasil. 2.ed. Nova Odessa: Plantarum, 1998. v.2. 352p.

NAPPO, M. E.; FONTES, M. A. L.; OLIVEIRAFILHO, A. T. Suficiência amostral e análise do tamanho de parcela para o estudo da regeneração natural do sub-bosque de povoamentos homogêneos de Mimosa scabrella Benth., em área minerada, em Poços de Caldas-MG. Revista Árvore, v.23, n.4, p.443-453, 1999.

NÓBREGA, A. M. F. Fitossociologia e aspectos de regeneração de remanescentes e repovoamentos florestais em várzea do rio MogiGuaçu, Luiz Antônio-SP. 2003. 159f. Tese (Doutorado em Agronomia) - Universidade Estadual Paulista, Jaboticabal, 2003.

OLIVEIRA, E. C. L.; FELFILI, J. M. Estrutura e dinâmica da regeneração natural de uma mata de galeria no Distrito Federal, Brasil. Acta Botânica Brasílica, v.19, n.4, p.801-811, 2005.

PASSOS, M. J. Estrutura da vegetação arbórea e regeneração natural em remanescentes de matas ciliares do rio Mogi-Guaçu - SP. 1998. 65f. Dissertação (Mestrado em Ciências Florestais) - Escola Superior de Agronomia “Luiz de Queiroz”, Piracicaba, 1998.
REZENDE, M. L. Regeneração natural de espécies florestais em sub-bosque de um povoamento de Eucalyptus grandis e de mata secundária, no município de Viçosa, Zona da Mata, Viçosa - MG. 1995. 116f. Dissertação (Mestrado em Ciências Florestais) - Universidade Federal de Viçosa, Viçosa, MG, 1995.

RODRIGUES, R. R. Restauração de florestas tropicais: indicadores de avaliação e monitoramento vegetal. In: SIMPÓSIO SOBRE RESTAURAÇÃO ECOLÓGICA DE ECOSSISTEMA NATURAIS, 1., 1999, Piracicaba. Anais...Piracicaba: Universidade de São Paulo/ESALQ/Departamento de Ciências Florestais, 1999. 8p.

SANTIAGO, J.; SILVA JÚNIOR, M. C.; LIMA, L. C. Fitossociologia da regeneração arbórea na mata de galeria do Pitoco (IBGE-DF), seis anos após fogo acidental. Scientia Forestalis, n.67, p.64-77, 2005.

SCARIOT, A. et al. Vegetação e flora. In: RAMBALDI, D. M.; OLIVEIRA, D. A. S. (Orgs.) Fragmentação de ecossistemas: causas, efeitos sobre a biodiversidade e recomendações de políticas públicas. Brasília: MMA/SBF, 2003.p.104-123.

SILVA, A. F. et al. Composição florística e grupos ecológicos das espécies de um trecho de floresta semidecídua submontana da Fazenda São Geraldo, Viçosa-MG. Revista Árvore, v.27, n.3, p.311-319, 2003.

SILVA JÚNIOR, W. M. Regeneração natural de espécies arbustivo-arbóreas em dois trechos de uma Floresta Estacional Semidecidual, Viçosa, MG. Scientia Forestalis, n.66, p.169-179, 2004.

SOUZA, V. C.; LORENZI, H. Botânica sistemática: guia ilustrado para identificação das famílias de Angiospermas da flora brasileira, baseado em APGII. Nova Odessa: Instituto Plantarum, 2005. 640p.

TABARELLI, M.; VILLANI, J. P.; MANTOVANI, W. Aspectos da sucessão secundária no Parque Estadual da Serra do Mar, SP. Revista Instituto Florestal, n.5, p.110-114, 1993. 